Somnologie $2018 \cdot 22: 227$

https://doi.org/10.1007/s11818-018-0184-2

(c) Springer Medizin Verlag GmbH, ein Teil von Springer Nature 2018

CrossMark

\title{
Helmut Frohnhofen
}

Fakultät für Gesundheit, Universität Witten/Herdecke, Witten, Deutschland

\section{Schlaf im hohen Lebensalter}

Ausreichender und qualitativguter Schlaf ist wichtig für Leistungsfähigkeit und Wohlbefinden. Das Schlafvermögen ändert sich mit zunehmendem Lebensalter. Angesichts des wachsenden Anteils alter Menschen an der Allgemeinbevölkerung steht auch die Schlafmedizin vor Herausforderungen. Neben einer verlässlichen und klaren schlafmedizinischen Diagnostik sind auch Behandlungskonzepte erforderlich, die aufgrund der funktionellen und kognitiven Probleme älterer Patienten ein Abweichen von den in der Schlafmedizin etablierten Standards erforderlich machen können.

Tagesschläfrigkeit ist ein häufiges Phänomen älterer Menschen. Die Ursache ist vielfältig. Die Abklärung stößt an Grenzen, da keines der in der Schlafmedizin verfügbaren Instrumente (Fragebögen) für beeinträchtigte ältere Menschen validiert wurde. Insbesondere die Epworth Sleepiness Scale als weltweit häufig verwendetes Instrument zeigt Schwächen bei der Anwendung in der Altersmedizin, wie Herr Dr. Frohnhofen in seinem Beitrag darlegt. Dies rechtfertigt die Entwicklung einer adaptierten Form zur Messung von Tagesschläfrigkeit bei älteren Menschen.

Eine besondere Gruppe alter Menschen sind die an Demenz Erkrankten. Diese Personengruppe zeigt immer Schlafstörungen. Dabei gibt es nicht eine spezifische Schlafstörung bei einer Demenz. Das Spektrum bleibt breit und macht eine differenzialdiagnostische Abklärung erforderlich. Nur so kann gezielt behandelt werden. Andererseits mehren sich die Hinweise darauf, dass gestörter Schlaf ein Risikofaktor für eine spätere Demenzentwicklung sein kann. Diese Zusammenhänge beschreibt Herr Prof. Hermann in seinem Beitrag. Für die Schlafmedizin ergibt sich hier die
Möglichkeit, einen Risikofaktor für eine Demenzentwicklung zu haben, der mit den Mitteln der Schlafmedizin potenziell modifizierbar ist.

Herr Prof. Netzer beschreibt in seinem Beitrag die Veränderungen des Schlafes mit dem Lebensalter. Er weist auf die alltagspraktischen Probleme bei der schlafmedizinischen Diagnostik und Therapie hin und benennt Bereiche, die weiter untersucht werden müssen.

Schlaflosigkeit ist ein häufiges Phänomen im hohen Lebensalter. Frau Prof. Richter zeigt, dass auch ältere Menschen von den verhaltenstherapeutischen Maßnahmen profitieren. Die Etablierung dieserErstlinientherapie sollte angesichts ihrer belegten Wirksamkeit und praktischen Nebenwirkungsfreiheit weiter gefördert werden.

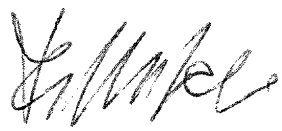

Dr. Helmut Frohnhofen

\section{Korrespondenzadresse}

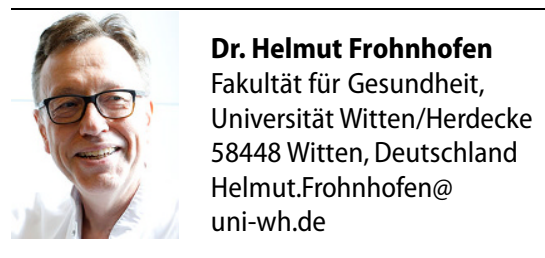

Interessenkonflikt. H. Frohnhofen gibt an, dass kein Interessenkonflikt besteht. 